\section{BS23 GENDER AND ASPIRIN IMPACT GENERATION OF PROCOAGULANT OXIDIZED PHOSPHOLIPIDS BY PLATELETS IN A HEALTHY HUMAN COHORT}

Majd Protty*, Christopher Brasher, Victoria Tyrrell, Patricia Rodrigues, Maceler Aldrovandi, Samya Obaji, P Vince Jenkins, Peter Collins, Valerie O’Donnell. Cardiff University

\subsection{6/heartjnl-2019-BCS.186}

Background Phospholipids on the surface of platelets play a key role in supporting coagulation in both health and disease. Alterations to phospholipid composition in platelets can contribute to thrombotic and bleeding disorders. We recently identified pro-coagulant enzymatically oxidized phospholipids (eoxPL) generated by platelets in response to thrombin activation via cyclooxygenase-1 (COX) and 12-lipoxygenase (LOX). It is not known how these lipids vary in relation to aspirin (a COX inhibitor) supplementation and/or gender.

Methods Twenty-eight healthy volunteers (14 males, 14 females) donated blood for platelet isolation at baseline (following a 14-day NSAID washout) and after 7-day supplementation with $75 \mathrm{mg}$ aspirin. Repeat sampling took place 2 months and 4 months later to account for variations over time. Lipids were extracted from platelets either basally or following $30 \mathrm{~min}$ thrombin activation then analysed using liquid chromatography with tandem mass-spectrometry (LC-MS/MS) for the 49 most abundant eoxPL molecular species.

Results The majority (96\%) of eoxPL increased in response to thrombin activation of platelets. Gender differences were observed with a trend towards higher levels generated by females. Correlation analysis highlighted clustering of eoxPL by enzymatic origin (12-LOX versus COX-1). Aspirin supplementation decreased eoxPL generated via COX-1 in both genders, but in males it increased levels of those generated by 12-LOX $(\mathrm{p}<0.05)$. Importantly, elevated levels of 12-hydroxyeicosatetraenoic acid (12-HETE)-containing eoxPL, which are known to be procoagulant, were observed. In contrast, generation of free 12-HETE was not affected by aspirin.

Conclusion In this study, we found an influence of both gender and aspirin supplementation on the generation of eoxPL in thrombin-activated platelets from healthy volunteers. Further characterisation of the gender influence may reveal novel mechanisms that contribute to the risk of arterial thrombosis in human populations.

Conflict of interest None declared

\section{BS24 PHARMACOKINETIC ANALYSIS AND EVALUATION OF APOCYNIN IN REDUCING CARDIAC SUPEROXIDE PRODUCTION}

Fangfei Liu*, Jian-Mei Li. Univeristy of Reading

10.1136/heartjnl-2019-BCS.187

Apocynin (4'-hydroxy-3'methoxyacetophenone) has been widely used in many preclinical and clinical studies as an inhibitor of the NADPH oxidase (Nox). However, its in vivo pharmacokinetics and tissue distribution has not been well evaluated. In this study, we investigated the in vivo PKPD of apocynin in samples of blood, heart, brain and urine. Apocynin was administered intravenously (i.v. bolus, $5 \mathrm{mg} / \mathrm{kg}$ ) to male CD1 mice (12 weeks of age), and its time-dependent tissue distribution was detected using a HPLC quadrupole- linear ion-trap tandem mass spectrometry (HPLC-MS). Plasma concentration of apocynin showed a mono-exponential decline pattern and reached $20646.56 \mathrm{ng} / \mathrm{ml}$ at $1 \mathrm{~min}$ after injection with a $t 1 / 2=2.85 \mathrm{~min}$ and a $\mathrm{CL}=1.77 \mathrm{ml} / \mathrm{min}$. It was also detected in urine at 15 min with a concentration of $3066.25 \mathrm{ng} / \mathrm{ml}$ and remained detectable until $60 \mathrm{~min}$ with a $C L R=0.0017 \mathrm{ml} / \mathrm{min}$. Apocynin was detected in cardiac tissues at a concentration of $1909.33 \mathrm{ng} / \mathrm{ml}$ at $1 \mathrm{~min}$ and remained detectable until $15 \mathrm{~min}$. Apocynin reached brain tissue $5 \mathrm{~min}$ of injection with a concentration of 1231.00 $\mathrm{ng} / \mathrm{ml}$ and lasted until $30 \mathrm{~min}$. The effect of apocynin to inhibit cardiac Nox2-derived O2.- production was investigated using cardiac homogenates of the same mice by lucigenin-chemiluminescence. Apocynin significantly reduced cardiac O2.- production at $15 \mathrm{~min}$ and returned to normal at $30 \mathrm{~min}$ post-injection with an IC50 $=589.79 \mathrm{ng} / \mathrm{ml}$ at 9.01 min. Angiotensin II (AngII)-induced cardiac O2.- production was reduced by $(59.81 \pm 20.23) \%$ by apocynin at a concentration of $100 \mu \mathrm{M}$ ex vivo, as compared to flavoprotein inhibitor, diphenyleneiodonium (DPI), which reduced O2.- by $(87.79 \pm 2.20) \%$. In conclusion, apocynin is able to reach cardiac tissues after i.v. bolus and effectively reduced cardiac O2.- production in mice.

Conflict of interest No

\section{BS25 INVESTIGATING THE MIR-101-3P/TRIB1 AXIS IN MACROPHAGE IMMUNOMETABOLISM}

Chiara Niespolo1* ${ }^{2,3}$ Juan Salamanca Viloria, ${ }^{4}$ Sumeet Deshmukh, ${ }^{2}$ Oscar Villacanas Perez, ${ }^{4}$ Ian Sudbery, ${ }^{1}$ Heather Wilson, ${ }^{1}$ Endre Kiss-Toth. 'Department of Infection, Immunity and Cardiovascular Disease, University of Sheffield; ${ }^{2}$ Mind the Byte (formerly Intelligent Pharma), Barcelona; ${ }^{3}$ University of Barcelona; ${ }^{4}$ Department of Molecular Biology and Biotechnology, University of Sheffield

\subsection{6/heartjnl-2019-BCS.188}

Introduction MiR-101-3p has been implicated in the regulation of macrophage cholesterol efflux and pro-inflammatory genes by directly targeting ABCA1 and MKP-1, which are both downregulated in atherosclerosis. By using bioinformatic analysis we identified TRIB1 as a direct target of miR-101. The TRIB1 gene plays major roles in myeloid cells, regulating inflammation and lipid metabolism. Its deficiency is associated with a severe reduction of anti-inflammatory tissue macrophages (M2-like cells) and an increase in plasma triglycerides.

Here we aimed to uncover the role of miR-101-3p/TRIB1 interaction and its consequences on human macrophage immunometabolism.

Methods MiRanda target prediction algorithm was used to identify macrophage-specific miRNAs targeting TRIB1, along with additional web-based tools. MiR-101-3p was selected based on its conservation among species as well as its high predictive score and free energy. A luciferase reporter assay was used to validate the miR-101-3p/TRIB1 interaction, employing a miR-101 mimic and inhibitor. Gene expression (RT-qPCR) and protein analysis (Western Blot) was carried out in human monocyte-derived macrophages (hMDMs) following transient manipulation of TRIB1 and miR-101 levels.

Results miR-101-3p has a functional binding site for the 3'UTR of TRIB1 and when overexpressed in hMDMs is able to reduce TRIB1 expression at both mRNA and protein levels. Over-expression of TRIB1 in hMDMs alters the levels of pro- 